\title{
Protective effects of curcumin on chromatin quality, sperm parameters, and apoptosis following testicular torsion-detorsion in mice
}

\author{
Abbas Shahedi ${ }^{1}$, Ali Reza Talebi ${ }^{1,2}$, Aghdas Mirjalili ${ }^{1}$, Majid Pourentezari ${ }^{1}$ \\ ${ }^{1}$ Department of Biology and Anatomical Sciences, ${ }^{2}$ Department of Reproductive Biology, Research and Clinical Center for Infertility, Shahid \\ Sadoughi University of Medical Sciences, Yazd, Iran
}

Objective: The chief outcome of testicular torsion in clinical and experimental contexts is testicular ischemia. Curcumin, a compound with anti-inflammatory and antioxidant properties, has fascinated researchers and clinicians for its promise in the treatment of fertility diseases. Methods: Thirty-five fully grown male mice were randomly classified into five groups: control, sham, testicular torsion, treatment group 1 (testicular torsion+short-term curcumin), and treatment group 2 (testicular torsion+long-term curcumin). Thirty-five days later, spermatozoa from the right cauda epididymis were analyzed with regard to count and motility. Toluidine blue (TB), aniline blue (AB), and chromomycin A3 (CMA3) staining assays were used to evaluate the sperm chromatin integrity. In addition, the terminal deoxynucleotidyl transferase-mediated deoxyuridine triphosphate nick-end labeling (TUNEL) test was used to assess apoptosis.

Results: Treatment group 1 exhibited a remarkably elevated sperm count compared to the testicular torsion group. Additionally, notably lower sperm motility was found in the testicular torsion group compared to the control, treatment 1, and treatment 2 groups. Staining (CMA3, AB, and TB) and the TUNEL test indicated significantly greater testicular torsion in the torsion group compared to the control group $(p<0.05)$. The data also revealed notably lower results of all sperm chromatin assays and lower apoptosis in both treatment groups relative to the testicular torsion group $(p<0.05)$. Significantly elevated $(p<0.05)$ AB and TB results were noted in treatment group 1 compared to treatment group 2.

Conclusion: Curcumin can compensate for the harmful effects of testicular ischemia and improve sperm chromatin quality in mice.

Keywords: Sperm; Spermatic cord torsion

\section{Introduction}

Infertility is a common problem that affects approximately $15 \%$ of couples who try to conceive [1]. In more than $50 \%$ of couples having difficulty conceiving, the problem is at least partially related to male reproductive issues [1,2]. A number of factors can cause male infertil-

Received: May 27, 2020 · Revised: September 10,2020 · Accepted:September 15, 2020 Corresponding author: Majid Pourentezari

Department of Biology and Anatomical Sciences, Shahid Sadoughi University of Medical Sciences, Alem Sq, Yazd 8916978477, Iran

Tel: +98-3538203410 Fax: +98-3538203414 E-mail:m.pourentezari@gmail.com

This is an Open Access article distributed under the terms of the Creative Commons Attribution Non-Commercial License (http://creativecommons.org/licenses/by-nc/4.0/) which permits unrestricted non-commercial use, distribution, and reproduction in any medium, provided the original work is properly cited. ity or subfertility, including testicular torsion [3], which is one of the most prevalent urological issues affecting young men. In 1988, Anderson and Williamson [4] reported the annual incidence of testicular torsion as 1 in 4,000 individuals younger than 25 years old. While this condition can occur at any age, it usually occurs in young males, with a bimodal incidence in the pediatric population: during the first year of life and between the ages of 13 and 16 years [2]. Therefore, a high rate of testicular torsion can be regarded as a crucial contributor to male infertility. Testicular torsion and torsion repair induce an ischemia-reperfusion injury to the testis that can render the testis aspermatogenic [5]. The injuries caused by testicular torsion are severe enough to lead to ipsilateral damage, which results from the significant increase in blood flow after detorsion [6]. A 90\% chance of res- 
cuing the testes exists if surgery is performed within the first 6 hours. This chance drops to $0 \%$ after 24 hours [7]. The treatment success rate is based on the duration of torsion and on early diagnosis as part of routine management for the preservation of spermatogenesis and fertility [8]. After the torsion event, the blood circulation is severed, which damages the tissue. After detorsion, blood flow is restored, which leads to events such as the production of reactive oxygen species (ROS) and reactive nitrogen species, lipid peroxidation, and the release of proinflammatory cytokines such as interleukin 6 and tumor necrosis factor alpha, resulting in further tissue injury [9]. Rapid reperfusion is vital for ischemic tissue survival and can limit the generation of oxygen-derived free radicals involved in pathophysiological cascades [10].

Since the 1940s, ROS have been presented in the literature as a potential contributor to male infertility [11]. Since then, major advances have been made in our understanding of the effects of ROS on infertility and sperm function. A recent study stated that ROS may be an important contributing factor affecting 30\%-80\% of infertile men [12]. Sperm cells are well-equipped against ROS attack with a robust defense mechanism involving antioxidants, but lack of balance between the production of ROS and the accessible antioxidant defenses results in oxidative stress [13]. A variety of antioxidants have been examined based on their capacity to either counteract ROS directly or impact the counter-ROS toxicity in the semen of different mammalian species.

Curcumin (1,7-bis[4-hydroxy-3-methoxyphenyl]-1,6-heptadiene-3,5-dione) is a major phytochemical commonly found in turmeric (Curcuma longa). In fact, the rhizome of the $C$. longa Linn plant contains curcumin. The role of curcumin as a free radical scavenger has been demonstrated in numerous studies $[14,15]$. Particularly in the testicular tissue, it can constrain the generation of ROS and nitric oxide in macrophages [16]. The antioxidant mechanism of curcumin comes from its specific conjugated structure, which includes the enol form of a $\beta$-diketone and two methoxylated phenols. This structure has the capability to trap free radicals as a chain-breaking antioxidant [17]. Remarkably, curcumin can prevent the generation of ROS both in vitro and in vivo. The associated improvement of sperm mobility and the decrease in spermatozoa defects have been claimed to potentially be due to the cessation of peroxidative alterations in the sperm and testicular membrane $[18,19]$.

In this regard, several in vivo and in vitro studies have emphasized the energy-promoting and protective properties of curcumin on male reproductive structures. In contrast, other reports have suggested that curcumin plays adverse roles in cellular signaling linked to spermatogenic processes $[20,21]$. This study therefore investigated the protective effects of curcumin on sperm count, chromatin condensation, motility, apoptosis, and DNA integrity following testic- ular torsion-detorsion in mice.

\section{Methods}

\section{Animals}

All animal experiments were approved by the Animal Ethics Committee at Shahid Sadoughi University of Medical Sciences (No. ir. ssumedicine.REC.1394412). Thirty-five adult NMRI mice 8-11 weeks in age and 35-40 $\mathrm{g}$ in weight were purchased from the Infertility Clinical Center of Shahid Sadoughi University of Medical Sciences in Yazd, Iran. During the experimental period, the mice were kept under standard laboratory conditions ( 12 hours: 12 hours light-dark cycle, $25^{\circ} \mathrm{C} \pm 2{ }^{\circ} \mathrm{C}$, and mean relative humidity of $50 \% \pm 5 \%$ ). They were fed with standard commercial laboratory chow and water. The mice were kept in separate cages for approximately 35 days (roughly equal to 1 period of spermatogenesis) [22].

\section{Experimental design}

The animals were randomly classified into five groups, each containing seven mice. The groups included (1) a control group, which was composed of mice that underwent no surgical procedure and were used for the determination of basal values of the parameters; (2) a sham group, which contained mice that underwent a sham operation for each surgical procedure, involving a midline scrotal incision and physical manipulation of the testis before placement back into the scrotum; (3) a testicular ischemia group; (4) treatment group 1 (testicular torsion+short-term curcumin), and (5) treatment group 2 (testicular torsion+long-term curcumin) [23].

The animals were first anesthetized via intraperitoneal injection of $100 \mathrm{mg} / \mathrm{kg}$ of ketamine and $10 \mathrm{mg} / \mathrm{kg}$ of xylazine, and an incision was made in the left inguinal region to access the testis. Then, after incising the scrotum, the left testis was extracted and rotated $720^{\circ}$ counterclockwise using a 0-6 suture thread to the scrotal wall. After 2 hours, the testicle was restored, and the surgical site was closed. In the testicular torsion+short-term curcumin group, a single $100 \mathrm{mg} / \mathrm{kg}$ dose of curcumin was injected intraperitoneally 30 minutes before reperfusion. In the testicular torsion+long-term curcumin group, curcumin was injected intraperitoneally at a daily dose of $100 \mathrm{mg} / \mathrm{kg}$ from 30 minutes before reproduction until day 35. All surgical procedures were performed under sterile conditions. After 35 days, the animals were anesthetized with ketamine and xylazine (150 mg/kg and $10 \mathrm{mg} / \mathrm{kg}$, respectively). One milliliter of prewarmed Ham F10 medium was added to the dissected cauda epididymis, and then delicate tearing was performed to allow the swim-out of spermatozoa into the culture medium. Finally, the dishes were incubated at $37^{\circ} \mathrm{C}$ and $5 \% \mathrm{CO}_{2}$ for 15 minutes. 


\section{Sperm analysis}

Sperm parameters, including count $\left(10^{6} / \mathrm{mL}\right)$ and motility, were assessed using 200 spermatozoa from each mouse. Sperm count and motility were investigated using a Makler chamber. Motility was stated as the percentages of rapid motility, slow motility, non-progressive motility, total motility, and immotile sperm.

\section{Sperm count}

The dissected epididymis from each mouse was transferred into 1 $\mathrm{mL}$ of Ham F10 medium and cut into small slices to allow swim-out of the spermatozoa into the medium. After 10 minutes of incubation, $10 \mu \mathrm{L}$ of the sample was used in a Makler chamber, and a light microscope (Olympus, Tokyo, Japan) was used to perform a sperm count based on World Health Organization guidelines. Data were stated as the number of spermatozoa per milliliter [24].

\section{Sperm motility}

Sperm motility evaluation was implemented in line with the World Health Organization protocol. In brief, $10 \mu \mathrm{L}$ of the sperm suspension was placed on a microscopic slide and a coverslip. At least 5 microscopic fields were investigated for each animal in order to assess sperm motility in a minimum of 200 spermatozoa [24].

\section{Sperm chromatin/DNA evaluation}

Standard cytochemical methods, including chromomycin A3 (CMA3), aniline blue (AB), and toluidine blue (TB), were used to assess chromatin condensation and DNA integrity. The dyes and chemicals used were obtained from Sigma-Aldrich (St. Louis, MO, USA). The dyes' effectiveness was carefully examined using standard samples with and without acid denaturation, which were reflected as positive and negative controls, respectively [25].

\section{1) $A B$ staining}

$A B$ selectively stains lysine-rich histones. This stain can also indicate anomalies in sperm chromatin condensation, which are germane to the residual histones. For this staining protocol, air-dried smears from the samples of washed semen were placed in $0.2 \mathrm{M}$ phosphate buffer ( $\mathrm{pH} 7.2$ ) containing $3 \%$ buffered glutaraldehyde for 30 minutes at room temperature. Each of the smears was stained in $4 \%$ acetic acid (pH 3.5) with $5 \%$ aqueous AB stain for 7 minutes. Under light microscopic assessment, 200 spermatozoa were meticulously counted in various parts of each slide with $\times 100$ eyepiece magnification [26].

\section{2) TB staining}

TB is a metachromatic dye. In sperm, it can be used as an indicator of the nuclear chromatin condensation and the DNA fragmentation quality and quantity by binding to the phosphate groups of DNA strands. In brief, $96 \%$ ethanol and acetone (1:1) were used to fix the air-dried sperm smears for 30 minutes at a temperature of $4^{\circ} \mathrm{C}$. The slides were then incubated in $0.1 \mathrm{~N} \mathrm{HCl}$ for 5 minutes at $4^{\circ} \mathrm{C}$. Afterwards, the slides were thoroughly washed with distilled water three times for 2 minutes each time. Ultimately, they were stained for 10 minutes at room temperature using $0.05 \%$ TB in 50\% citrate phosphate. In each sample, a minimum of 200 spermatozoa were counted using light microscopy with $\times 100$ eyepiece magnification [26].

\section{3) CMA3 staining}

CMA 3 (Sigma, St. Louis, MO, USA) is a fluorochrome. It is used specifically for the detection of cytosine-rich and guanosine sequences and the evaluation of the degree of protamination of the chromatin in sperm. In this procedure, the sperm cells were fixed at $48^{\circ} \mathrm{C}$ for 10 minutes in Carnoy solution, which contains a 3 to 1 ratio of methanol:glacial acetic acid. Then, the slides were stained with CMA3 solution $(0.25 \mathrm{mg} / \mathrm{mL}$ in Mcllvaine buffer; $7 \mathrm{~mL}$ of $0.1 \mathrm{M}$ citric acid+32.9 $\mathrm{mL}$ of $0.2 \mathrm{M} \mathrm{Na}_{2} \mathrm{HPO}_{4} \cdot 7 \mathrm{H}_{2} \mathrm{O}, \mathrm{pH} 7.0$, containing $10 \mathrm{mM} \mathrm{MgCl}$ ) for 20 minutes in a dark room. A fluorescence microscope (Olympus BX51) was used to count a minimum of 200 spermatozoa with a filter of $460 \mathrm{~nm}$ and $\times 100$ eyepiece magnification [27].

\section{Terminal deoxynucleotidyl transferase-mediated deoxyuridine triphosphate nick-end labeling assay}

The terminal deoxynucleotidyl transferase-mediated deoxyuridine triphosphate nick-end labeling (TUNEL) test is based on the detection of single- or double-stranded DNA fragments marked by dUTP due to a reaction catalyzed by the TdT enzyme. The TUNEL test generally consists of the fixation and permeabilization of sperm, the marking of DNA fragments, staining, and the evaluation of the stages of sperm. In the evaluation process, the sperm can be assessed subjectively under light and fluorescence microscopy or by flow cytometry [28]. The TUNEL assay can also be used to identify apoptotic cells in the tissues during histological examinations.

In this study, the TUNEL assay was used to detect DNA fragmentation in the spermatozoa. This was done utilizing an in situ cell death detection kit along with tetramethylrhodamine red-labeled dUTP (Roche Diagnostics, Basel, Switzerland). To determine the TUNEL-positive spermatozoa proportion, as previously described, a fluorescence microscope (Olympus BX50) was used for the final assessment. Using the appropriate filter, at least 200 spermatozoa per slide were counted [29].

\section{Statistical analysis}

The data were stated as mean \pm standard deviation. One-way analysis of variance was used to evaluate the significance of the dif- 
ferences among the four groups, and the Tukey post hoc test was performed to determine the differences between pairs of groups. Statistical significance was indicated by a two-sided $p$-value $<0.05$ for the sperm parameters and cytochemical tests.

\section{Results}

Table 1 presents the sperm parameters of the five groups. This table demonstrates that for the majority of the sperm parameters, sig- nificant differences $(p<0.05)$ were present between different groups. Rapid motility and total motility were significantly lower in the testicular torsion group than in the control and sham groups. Significantly greater total motility was also noted in the treatment 1 and treatment 2 groups compared to the testicular torsion group, and significantly greater rapid motility was observed in the treatment 2 group compared to the testicular torsion group.

Table 2 presents the data on sperm chromatin condensation, DNA integrity, and apoptosis. Statistically significant differences $(p<0.05)$

Table 1. Results of sperm parameters analysis by group

\begin{tabular}{|c|c|c|c|c|c|c|}
\hline Variable & Control & Sham & Testicular torsion & Treatment 1 & Treatment 2 & $p$-value \\
\hline Count $\left(\times 10^{6}\right)$ & $18.33 \pm 1.66$ & $16.66 \pm 3.07$ & $8.33 \pm 1.05$ & $21.66 \pm 5.01$ & $15.83 \pm 2.71$ & $0.015^{\mathrm{d})}$ \\
\hline \multirow[t]{3}{*}{ Rapid motility (\%) } & $25 \pm 2.88$ & $25 \pm 3.65$ & $0.83 \pm 0.83$ & $7.5 \pm 2.14$ & $15 \pm 3.65$ & $0.000^{\mathrm{a})}$ \\
\hline & & & & & & $0.002^{\mathrm{b})}$ \\
\hline & & & & & & $0.013^{\mathrm{e})}$ \\
\hline \multirow[t]{2}{*}{ Slow motility (\%) } & $24.16 \pm 3$ & $21.66 \pm 1.66$ & $5 \pm 1.29$ & $15.83 \pm 3$ & $20.83 \pm 1.53$ & $0.000^{\mathrm{a}, \mathrm{e})}$ \\
\hline & & & & & & $0.016^{\mathrm{d})}$ \\
\hline \multirow[t]{3}{*}{ Non-progressive motility (\%) } & $18.33 \pm 1.66$ & $16.66 \pm 3.07$ & $8.33 \pm 1.05$ & $21.66 \pm 4.01$ & $50.83 \pm 2$ & $0.017^{c)}$ \\
\hline & & & & & & $0.01^{\mathrm{d})}$ \\
\hline & & & & & & $0.002^{f)}$ \\
\hline \multirow[t]{5}{*}{ Immotile sperm (\%) } & $32.5 \pm 4.23$ & $36.66 \pm 3.57$ & $85.83 \pm 2.38$ & $55 \pm 8.85$ & $58.33 \pm 4.4$ & $0.000^{\mathrm{a})}$ \\
\hline & & & & & & $0.037^{b)}$ \\
\hline & & & & & & $0.013^{c)}$ \\
\hline & & & & & & $0.002^{\mathrm{d})}$ \\
\hline & & & & & & $0.008^{\mathrm{e})}$ \\
\hline \multirow[t]{5}{*}{ Total motility } & $67.5 \pm 4.23$ & $67.5 \pm 2.81$ & $14.16 \pm 2.38$ & $43.33 \pm 8.02$ & $41.66 \pm 4.4$ & $0.000^{\mathrm{a})}$ \\
\hline & & & & & & $0.012^{b)}$ \\
\hline & & & & & & $0.007^{c)}$ \\
\hline & & & & & & $0.002^{\mathrm{d})}$ \\
\hline & & & & & & $0.004^{\mathrm{e})}$ \\
\hline
\end{tabular}

Values are presented as mean \pm standard deviation.

${ }^{\mathrm{a})}$ Difference between control and testicular torsion group; ${ }^{\mathrm{b})}$ Difference between control and treatment 1 group; ${ }^{\mathrm{c}}$ Difference between control and treatment 2 group; ${ }^{\mathrm{d})}$ Difference between testicular torsion and treatment $1 \mathrm{group} ;{ }^{\mathrm{e})}$ Difference between testicular torsion and treatment 2 group; ${ }^{\mathrm{f})}$ Difference between treatment 1 group and treatment 2 group.

Table 2. Results of sperm chromatin/DNA integrity and apoptosis in different groups

\begin{tabular}{|c|c|c|c|c|c|c|}
\hline Variable & Control & Sham & Testicular torsion & Treatment 1 & Treatment 2 & $p$-value \\
\hline CMA3 & $2.83 \pm 0.6$ & $3 \pm 0.51$ & $13.66 \pm 0.66$ & $7.66 \pm 0.49$ & $5.83 \pm 0.77$ & $\begin{array}{l}0.000^{\mathrm{a}, \mathrm{b}, \mathrm{d}, \mathrm{e})} \\
0.006^{\mathrm{c})}\end{array}$ \\
\hline$A B$ & $3.16 \pm 1.07$ & $3 \pm 1.03$ & $36.33 \pm 2.75$ & $20.33 \pm 2.77$ & $5.16 \pm 0.79$ & $0.000^{a, b, d, e, f)}$ \\
\hline TB & $3.33 \pm 1.08$ & $5.83 \pm 1.55$ & $36 \pm 3.38$ & $20.83 \pm 3.31$ & $8.66 \pm 2.38$ & $\begin{array}{l}0.000^{\mathrm{a}, \mathrm{b}, \mathrm{e})} \\
0.002^{\mathrm{d})} \\
0.017^{\mathrm{f})}\end{array}$ \\
\hline TUNEL & $1.33 \pm 0.21$ & $1.5 \pm 0.22$ & $22.66 \pm 3.09$ & $9.33 \pm 0.71$ & $7.33 \pm 1.57$ & $\begin{array}{l}0.000^{\mathrm{a}, \mathrm{d}, \mathrm{e})} \\
0.006^{\mathrm{b})}\end{array}$ \\
\hline
\end{tabular}

Values are presented as mean \pm standard deviation.

CMA3, chromomycin A3; AB, aniline blue; TB, toluidine blue; TUNEL, terminal deoxynucleotidyl transferase-mediated deoxyuridine triphosphate nick-end labeling.

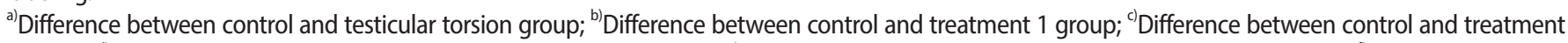
2 group; ${ }^{\mathrm{d})}$ Difference between testicular torsion and treatment $1 \mathrm{group} ;{ }^{\mathrm{e})}$ Difference between testicular torsion and treatment $2 \mathrm{group} ;{ }^{\mathrm{f})}$ Difference between treatment 1 group and treatment 2 group. 
present between different groups are indicated. Sperm chromatin damage and apoptosis were observed to increase after testicular torsion. However, in the curcumin groups (treatment groups 1 and 2), the extent of damage was lower than in the testicular torsion group, with particularly remarkable results in the treatment 2 group.

\section{Discussion}

The present study was intended to investigate the effects of curcumin on sperm damage in animals after exposure to testicular torsion. Testicular torsion, which is associated with severe scrotal pain and ischemia, is an emergent pathologic state requiring immediate surgery [30]. A few hours of testicular torsion may harm the testicle and can sometimes necessitate the removal of the impaired testicles [31]. After the surgical procedure, it may not be apparent whether the function of the testes has been entirely preserved. Therefore, finding new strategies and therapeutic applications to serve as adjuvant therapies to surgical detorsion seems necessary [3]. Surgery is aimed to restore blood flow and correct the perfusion of the testis. After surgical detorsion, overproduction of free radicals such as ROS and overexpression of proinflammatory cytokines, such as interleukin 1-beta and tumor necrosis factor alpha, occurs. Proinflammatory cytokines are responsible for the recruitment of neutrophils to the testicular tissue [5]. The disruption of spermatogenesis can be triggered by ischemic reperfusion injury on testicular torsion, as demonstrated by lkebuaso et al [32]. Those researchers also showed that the short-term suppression of the secretion of testosterone could be permanent. The adverse effects of certain levels of ROS vary across semen samples. Sperm vulnerability, which varies among individuals and is related to the integrity of the sperm chromatin, may explain the occurrence of severe ROS sequelae [33].

Moreover, the effect of testicular torsion on DNA damage via the induction of oxidative stress and the generation of ROS has been demonstrated [34]. According to numerous studies, ischemia-reperfusion of the testis initiates several mechanisms, including the activation of inflammatory mediators such as cytokines, the infiltration of neutrophils, and the production of $\mathrm{NO}$ and ROS, damaging the cell membrane and DNA [3,35]. Our study also showed that testicular torsion decreased the parameters and the quality of sperm chromatin compared to the control group.

Sperm DNA integrity is an indispensable factor involved in the success of fertilization as well as in the healthy development of the embryo [36]. The molecular mechanism by which curcumin aids in proper sperm function remains unknown; however, the trapping of free radicals using $\beta$-diketone, methoxy, and phenolic functional groups is an antioxidant mechanism by which curcumin likely enhances sperm parameters [36].
Curcumin inhibits the generation of superoxide anions and hydroxyl radicals by preventing the oxidation of $\mathrm{Fe}^{2+}$ and $\mathrm{Fe}^{3+}$ through the Fenton reaction. However, another reason for the improvement of sperm motility may be the increase in the concentrations of ROS-scavenging molecules as a result of supplementation with curcumin [37]. Curcumin can also have preventive effects on oxidative stress via the suppression of nuclear factor-KB (NF-KB) DNA-binding activity and, by extension, of the expression of genes that require NF$\mathrm{KB}$ for their activation [38]. Some reports in the literature claim that curcumin improves the sperm parameters and DNA integrity $[13,18,19]$. Our results seem to corroborate this. We found that curcumin had a beneficial effect on sperm count, motility, sperm chromatin condensation, and apoptosis in mice subjected to testicular torsion, especially over an extended period of supplementation. This result supports the potential application of curcumin in boosting sperm quality. Many studies have reported that curcumin decreases malondialdehyde levels and oxidative stress in various tissues [39]. Thus, it can be assumed that curcumin, in its antioxidant capacity, inhibits ROS and induces antioxidant responses in the cell and thereby reduces the adverse effects of testicular torsion and mitigates sperm damage.

An ischemia-reperfusion injury to the testis may be induced by testicular torsion followed by torsion repair, and such an injury can impact the spermatogenic capacity of the testis. Study results have shown that the loss of spermatogenic capacity may be due to germ cell apoptosis induced by oxidative stress [5]. Previous reports have demonstrated the occurrence of damage after testicular torsion repair, most likely due to germ cell apoptosis and severe seminiferous impairment. Our data showed that curcumin could reduce sperm apoptosis in a group of mice affected by testicular torsion. In 2018, Zha et al. [40] claimed that curcumin reduced testicular apoptosis caused by diabetes by "regulating apoptotic proteins and markedly inhibiting oxidative stress levels by downregulating malondialdehyde expression, decreasing NADPH activity, and restoring antioxidant enzymes". Wei et al. [16] conducted a study on the effect of curcumin on ischemia-reperfusion injury in rat testes by rotating the left testicle $720^{\circ}$ for 2 hours after opening the scrotum and providing curcumin to the treatment group intravenously. The results showed that testicular torsion-detorsion significantly increased xanthine oxidase activity and malondialdehyde levels. In contrast, the group treated with curcumin exhibited reduced xanthine oxidase activity and malondialdehyde levels [16]. However, in our study, we focused on the parameters and DNA quality of sperm.

This study reported the deleterious effects of testicular torsion on sperm parameters and sperm chromatin quality. Curcumin can not only compensate for the destructive effects of testicular ischemia, but can also improve sperm chromatin quality in mice. In other 
words, the administration of curcumin after reperfusion surgery in mice played a protective role in cases of testicular torsion. Moreover, ischemia/reperfusion sperm injury was reduced through the decrease of oxidative stress and reduction in anti-inflammatory agents. Other advantages of curcumin are its inexpensiveness and its availability under typical conditions.

\section{Conflict of interest}

No potential conflict of interest relevant to this article was reported.

\section{ORCID}

Abbas Shahedi
Ali Reza Talebi
Aghdas Mirjalili
Majid Pourentezari

\section{Author contributions}

Conceptualization: ART, AS, MP. Data curation: AM. Formal analysis: AS, MP, ART. Methodology: all authors. Project administration: ART. Visualization: AS. Writing-original draft: AS, MP, AM. Writing-review \& editing: all authors.

\section{References}

1. Schilling K, Toth B, Rosner S, Strowitzki T, Wischmann T. Prevalence of behaviour-related fertility disorders in a clinical sample: results of a pilot study. Arch Gynecol Obstet 2012;286:1307-14.

2. Azizollahi S, Aflatoonian R, Sadighi Gilani MA, Behnam B, Tajik N, Asghari-Jafarabadi M, et al. Alteration of spermatogenesis following spermatogonial stem cells transplantation in testicular torsion-detorsion mice. J Assist Reprod Genet 2016;33:771-81.

3. Yousefi-Manesh H, Shirooie S, Hemati S, Shokrian-Zeini M, Zarei N, Raoufi M, et al. Protective effects of modafinil administration on testicular torsion/detorsion damage in rats. Exp Mol Pathol 2019;111:104305.

4. Anderson JB, Williamson RC. Testicular torsion in Bristol: a 25-year review. Br J Surg 1988;75:988-92.

5. Turner TT, Lysiak JJ, Shannon JD, Nguyen QA, Bazemore-Walker CR. Testicular torsion alters the presence of specific proteins in the mouse testis as well as the phosphorylation status of specific proteins. J Androl 2006;27:285-93.

6. Nguyen L, Lievano G, Ghosh L, Radhakrishnan J, Fornell L, John E. Effect of unilateral testicular torsion on blood flow and histology of contralateral testes. J Pediatr Surg 1999;34:680-3.

7. Mellick LB, Sinex JE, Gibson RW, Mears K. A systematic review of testicle survival time after a torsion event. Pediatr Emerg Care 2019;35:821-5.

8. Wampler SM, Llanes M. Common scrotal and testicular problems. Prim Care 2010;37:613-26.

9. Shimizu S, Tsounapi P, Dimitriadis F, Higashi Y, Shimizu T, Saito M. Testicular torsion-detorsion and potential therapeutic treatments: a possible role for ischemic postconditioning. Int J Urol 2016; 23:454-63.

10. Filho DW, Torres MA, Bordin AL, Crezcynski-Pasa TB, Boveris A. Spermatic cord torsion, reactive oxygen and nitrogen species and ischemia-reperfusion injury. Mol Aspects Med 2004;25:199-210.

11. MacLeod J. The role of oxygen in the metabolism and motility of human spermatozoa. Am J Physiol Legacy Content 1943;138: $512-8$.

12. Wagner H, Cheng JW, Ko EY. Role of reactive oxygen species in male infertility: an updated review of literature. Arab J Urol 2017; 16:35-43.

13. Soleimanzadeh A, Saberivand A. Effect of curcumin on rat sperm morphology after the freeze-thawing process. Vet Res Forum 2013;4:185-9.

14. Lee WH, Loo CY, Bebawy M, Luk F, Mason RS, Rohanizadeh R. Curcumin and its derivatives: their application in neuropharmacology and neuroscience in the 21st century. Curr Neuropharmacol 2013;11:338-78.

15. Wal P, Saraswat N, Pal RS, Wal A, Chaubey M. A detailed insight of the anti-inflammatory effects of curcumin with the assessment of parameters, sources of ROS and associated mechanisms. Open Med J 2019;6:64-76.

16. Wei SM, Yan ZZ, Zhou J. Curcumin attenuates ischemia-reperfusion injury in rat testis. Fertil Steril 2009;91:271-7.

17. Masuda T, Maekawa T, Hidaka K, Bando H, Takeda Y, Yamaguchi H. Chemical studies on antioxidant mechanism of curcumin: analysis of oxidative coupling products from curcumin and linoleate. J Agric Food Chem 2001;49:2539-47.

18. Kazemizadeh A, Zare Shahneh A, Zeinoaldini S, Yousefi AR, Mehrabani Yeganeh $\mathrm{H}$, Ansari Pirsaraei Z, et al. Effects of dietary curcumin supplementation on seminal quality indices and fertility rate in broiler breeder roosters. Br Poult Sci 2019;60:256-64.

19. Zhang L, Diao RY, Duan YG, Yi TH, Cai ZM. In vitro antioxidant effect of curcumin on human sperm quality in leucocytospermia. Andrologia 2017;49:e12760.

20. Naz RK. Can curcumin provide an ideal contraceptive? Mol Reprod Dev 2011;78:116-23.

21. Xia X, Cai H, Qin S, Xu C. Histone acetylase inhibitor curcumin impairs mouse spermiogenesis: an in vitro study. PLoS One 2012;7: 
e48673.

22. Ray D, Pitts PB, Hogarth CA, Whitmore LS, Griswold MD, Ye P. Computer simulations of the mouse spermatogenic cycle. Biol Open 2014:4:1-12.

23. Sharma P, Aslam Khan I, Singh R. Curcumin and quercetin ameliorated cypermethrin and deltamethrin-induced reproductive system impairment in male Wistar rats by upregulating the activity of pituitary-gonadal hormones and steroidogenic enzymes. Int J Fertil Steril 2018;12:72-80.

24. Pourentezari M, Talebi AR, Mangoli E, Anvari M, Rahimipour M. Additional deleterious effects of alcohol consumption on sperm parameters and DNA integrity in diabetic mice. Andrologia 2016; 48:564-9.

25. Talebi AR, Sarcheshmeh AA, Khalili MA, Tabibnejad N. Effects of ethanol consumption on chromatin condensation and DNA integrity of epididymal spermatozoa in rat. Alcohol 2011;45:403-9.

26. Pourmasumi S, Khoradmehr A, Rahiminia T, Sabeti P, Talebi AR, Ghasemzadeh J. Evaluation of sperm chromatin integrity using aniline blue and toluidine blue staining in infertile and normozoospermic men. J Reprod Infertil 2019;20:95-101.

27. Rahiminia T, Yazd EF, Fesahat F, Moein MR, Mirjalili AM, Talebi AR. Sperm chromatin and DNA integrity, methyltransferase mRNA levels, and global DNA methylation in oligoasthenoteratozoospermia. Clin Exp Reprod Med 2018;45:17-24.

28. Kucuk N. Sperm DNA and detection of DNA fragmentations in sperm. Turk J Urol 2018;44:1-5.

29. Petersen CG, Mauri AL, Vagnini LD, Renzi A, Petersen B, Mattila M, et al. The effects of male age on sperm DNA damage: an evaluation of 2,178 semen samples. JBRA Assist Reprod 2018;22:32330.

30. Kostakis ID, Zavras N, Damaskos C, Sakellariou S, Korkolopoulou P, Misiakos EP, et al. Erythropoietin and sildenafil protect against ischemia/reperfusion injury following testicular torsion in adult rats. Exp Ther Med 2017;13:3341-7.

31. Ghasemnejad-Berenji M, Ghazi-Khansari M, Pashapour S, Jafari A,
Yazdani I, Ghasemnejad-Berenji H, et al. Synergistic effect of rapamycin and metformin against germ cell apoptosis and oxidative stress after testicular torsion/detorsion-induced ischemia/reperfusion in rats. Biomed Pharmacother 2018;105:645-51.

32. Ikebuaso AD, Yama OE, Duru FI, Oyebadejo SA. Experimental testicular torsion in a rat model: effects of treatment with Pausinystalia macroceras on testis functions. J Reprod Infertil 2012; $13: 218-24$.

33. Agarwal A, Cho CL, Esteves SC, Majzoub A. Reactive oxygen species and sperm DNA fragmentation. Transl Androl Urol 2017; 6(Suppl 4):S695-6.

34. DaJusta DG, Granberg CF, Villanueva C, Baker LA. Contemporary review of testicular torsion: new concepts, emerging technologies and potential therapeutics. J Pediatr Urol 2013;9(6 Pt A):72330

35. Granger DN, Kvietys PR. Reperfusion injury and reactive oxygen species: the evolution of a concept. Redox Biol 2015;6:524-51.

36. Kim SM, Kim SK, Jee BC, Kim SH. Effect of sperm DNA fragmentation on embryo quality in normal responder women in in vitro fertilization and intracytoplasmic sperm injection. Yonsei Med J 2019;60:461-6

37. Aparnak P, Saberivand A. Effects of curcumin on canine semen parameters and expression of NOX5 gene in cryopreserved spermatozoa. Vet Res Forum 2019;10:221-6.

38. Singh S, Aggarwal BB. Activation of transcription factor NF-kappa $B$ is suppressed by curcumin (diferuloylmethane) [corrected]. J Biol Chem 1995;270:24995-5000.

39. Khalaji N, Namyari M, Rasmi Y, Pourjabali M, Chodari L. Protective effect of curcumin on fertility of rats after exposure to compact fluorescent lamps: an experimental study. Int J Reprod Biomed 2018;16:447-54.

40. Zha W, Bai Y, Xu L, Liu Y, Yang Z, Gao H, et al. Curcumin attenuates testicular injury in rats with streptozotocin-induced diabetes. Biomed Res Int 2018;2018:7468019. 